\title{
PARTICLE-GENERATED TURBULENCE IN HOMOGENEOUS DILUTE DISPERSED FLOWS
}

\author{
M. Mizukami, $\dagger$ R. N. Parthasarathy $\ddagger$ and G. M. Faeth \\ Department of Aerospace Engineering, University of Michigan, Ann Arbor, MI 48109-2140, U.S.A.
}

(Received 6 June 1991; in revised form 15 December 1991)

\begin{abstract}
Homogeneous turbulence generated by uniform fluxes of round glass beads $(0.5,1.0$ and $2.0 \mathrm{~mm}$ dia) falling through stagnant (in the mean) air was studied for particle Reynolds numbers in the range $100-800$ and particle volume fractions $<0.0004 \%$. Moments, probability density functions, spatial correlations and temporal spectra of air velocity fluctuations were measured using two-point phasediscriminating laser velocimetry. Predictions based on a simplified stochastic analysis, involving linear superposition of randomly-arriving particle velocity fields, were used to help interpret the measurements. Guided by the theory, correlations of turbulence properties were achieved for both the present particle/air and earlier particle/water measurements. Turbulence intensities (referenced to mean particle relative velocities) and integral scales are functions of the rate of dissipation of particle mechanical energy and particle drag properties; however, normalized probability density functions, spatial correlations and temporal spectra are largely independent of particle properties.
\end{abstract}

Key Words: dispersed flow, turbulence generation, homogeneous turbulence

\section{INTRODUCTION}

The objective of this investigation was to study the influence of turbulence/dispersed-phase interactions on the continuous-phase turbulence properties of dispersed multiphase flows. These interactions are important in either dense or homogeneous dispersed flows-homogeneous dilute dispersed flows were studied because they are more tractable for measurements. Experimental conditions involved uniform fluxes of particles falling in nearly stagnant (in the mean) air, in order to supplement earlier findings for similar particle/water flows (Parthasarathy \& Faeth 1990). A simplified model of the flow, developed by Parthasarathy \& Faeth (1990), was used to help interpret and correlate both sets of measurements.

Turbulence modulation and generation are two important turbulence/dispersed-phase interactions that affect continuous-phase turbulence properties in dispersed multiphase flows (Hinze 1972). Turbulence modulation is an interaction between continuous- and dispersed-phase velocity fluctuations which supplements conventional damping of continuous-phase turbulence (Al Tawell \& Landau 1977); it is most important when relative turbulence intensities (continuousphase r.m.s. velocity fluctuations normalized by the mean relative velocity between the phases) are large, and the dispersed-phase is responsive to continuous-phase velocity fluctuations. This mechanism is often included in turbulence models that allow for the effects of separated flow, where it appears as a damping term when the governing equation for continuous-phase turbulence kinetic energy is derived. However, methods of modeling turbulence modulation have not been definitively assessed because the phenomenon is most important for dense dispersed flows within the Stokes drag regime where accurate measurements are problematical (Faeth 1987).

Turbulence generation involves perturbation of the continuous-phase flow by the wakes of individual dispersed-phase elements. Turbulence generation in dispersed flows is somewhat similar to the action of turbulence-generating grids, however, there are significant differences between the properties of the two flows because dispersed-phase elements are distributed throughout the flow and their arrival at any point is random. Turbulence generation is most important when velocity defects within the wakes of dispersed-phase elements are large in comparison to background continuous-phase velocity fluctuations, which requires large relative velocities between the phases

†, $¥$ Present addresses: †Propulsion Systems Division, NASA Lewis Research Center, Cleveland, Ohio, U.S.A.; $¥ I n s t i t u t e ~ o f$ Hydraulic Research, University of Iowa, Iowa City, Iowa, U.S.A. 
and correspondingly low relative turbulence intensities. Additionally, the effects of turbulence generation are most significant in nearly homogeneous flows where other sources of turbulence production are weak. Thus turbulence generation in practical multiphase flows, evidenced by increased anisotropy and turbulence levels in comparison to corresponding single-phase flows, has been observed in the near-axis regions of turbulent bubbly and particle-laden jets (Sun \& Faeth 1986; Parthasarathy \& Faeth 1987). Similar flow conditions exist within the near-injector (dense-spray) region of sprays, the spray-containing region of large liquid rocket engines, and heavy rainstorms, among others. Motivated by these applications, the present investigation concentrates on the properties of turbulence generation.

Past experimental investigations of turbulence generation have considered homogeneous dilute dispersed flows (Lance \& Bataille 1982; Lance et al. 1980, 1985; Parthasarathy \& Faeth 1990). Lance and coworkers studied homogeneous air/water bubbly flows downstream of a turbulence-generating grid. The effects of turbulence generation were observed as a progressive increase in continuous-phase turbulence levels with increasing void fractions, with the increase being most evident when relative velocities were comparable to liquid velocities. These results are valuable because most practical multiphase flows involve turbulence generation by the dispersed phase as well as other forms of turbulence production in the continuous phase. However, the results are difficult to interpret, due to the combined effects of bubble- and grid-generated turbulence.

Earlier work in this laboratory considered flows where turbulence generation was the dominant mechanism of turbulence production (Parthasarathy \& Faeth 1990). The experimental conditions consisted of a uniform number fluxes of nearly monodisperse round glass beads falling at their terminal velocities in stagnant (in the mean) water. The flows were dilute (particle volume fractions $<0.01 \%$ ) and the effects of turbulence modulation were small (relative turbulence intensities $<10 \%$ ). Measurements included phase velocities and the probability density functions, temporal spectra and spatial correlations of liquid velocity fluctuations. Liquid-phase properties also were analyzed using a simplified stochastic method that involved linear superposition of randomly-arriving particle velocity fields. It was found that continuous-phase turbulence levels were largely controlled by the rate of dissipation of particle energy in the liquid. In contrast to grid-generated turbulence far from the source, where the turbulence is nearly isotropic, streamwise velocity fluctuations were nearly twice the cross-stream velocity fluctuations, which suggested a significant direct contribution of particle wakes to the observed properties of the continuous phase. The temporal spectra also supported this view: they exhibited a large range of scales even though particle Reynolds numbers were low $\left(<10^{3}\right)$, and they decayed at slower rates than conventional turbulence with increasing frequency. These features agreed with predictions based on superposition of the mean velocity profiles of randomly-arriving particle wakes. However, while the theory assisted interpretation of flow properties, quantitative predictions of integral scales and spatial correlations were not very satisfactory and there were convergence problems similar to those encountered when sedimentation is treated using stochastic methods (Batchelor 1972). Thus, generalization of the particle/water results to other flow conditions is questionable due to a limited range of experimental conditions and uncertainties of the theory.

The objective of the present investigation was to extend the particle/water study to particle/air flows. The primary motivation for this step is that rates of dissipation of particle energy in air are orders of magnitude larger than for particles in water at similar conditions so that effects of this important parameter can be resolved. Particle/air flows also reduce complications due to turbulent dispersion of particles because the particle response is smaller in air than in water. Other aspects of the present study are similar to Parthasarathy \& Faeth (1990); in particular, particle Reynolds numbers for both studies were $<10^{3}$ because this range is typical of drops in sprays and rainstorms (Faeth 1987; Humphreys 1964).

The paper begins with a description of the experimental and theoretical methods. Measured and predicted results are then considered, treating evaluation of the apparatus, velocity fluctuations, spatial correlations and temporal spectra in turn. Additional details about the experimental and theoretical methods can be found in Parthasarathy (1989) and Parthasarathy \& Faeth (1990). 


\section{EXPERIMENTAL METHODS}

\subsection{Apparatus}

Figure 1 is a sketch of the particle/air flow apparatus. The tests were conducted using nearly monodisperse spherical glass particles having nominal diameters of $0.5,1.0$ and $2.0 \mathrm{~mm}$. The flow of particles was controlled by a variable-speed particle feeder, whose feed rate was calibrated by collecting particles for timed intervals. The particles were dispersed by falling through an array of six screens $(0.89 \mathrm{~mm}$ dia and spaced $4.2 \mathrm{~mm}$ apart) with a $190 \mathrm{~mm}$ spacing between screens. The particles then fell into a windowed test chamber $(410 \times 535 \times 910 \mathrm{~mm})$, where the measurements were made. The particles were collected, with little rebound, at the bottom of the tank and removed periodically so that their maximum depth was $100 \mathrm{~mm}$. Displacement velocities due to the collection of particles at the bottom of the tank were negligible, $<0.02 \mathrm{~mm} / \mathrm{s}$.

The free fall distance of the particles between the last screen and the region where measurements were made was $1200 \mathrm{~mm}$, which was not sufficient for the particles to reach their terminal velocities. However, the rate of increase of particle velocities within the region of observations was small $(15-35 \% / \mathrm{m}$ or $2-4 \% /$ streamwise integral length scale) so that the flow was nearly homogeneous in the streamwise direction. Air velocities within the flow were small (ca. $10 \mathrm{~mm} / \mathrm{s}$; therefore, drafts and natural convection disturbances were controlled by closing all joints with tape and covering the exterior surfaces of the apparatus with styrofoam insulation (not shown in figure 1) except for small openings needed for optical access.

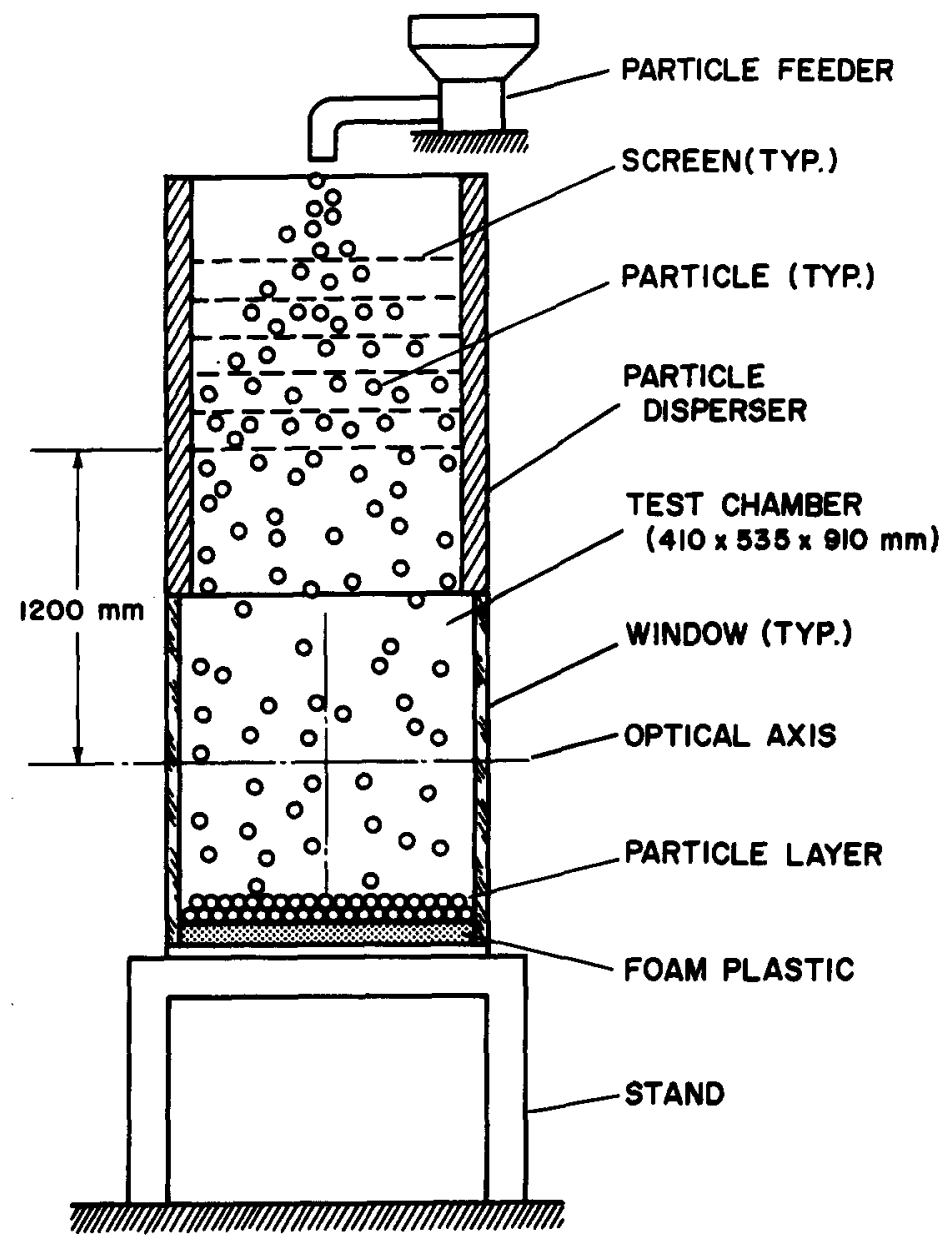

Figure 1. Sketch of homogeneous particle/air flow apparatus. 


\subsection{Instrumenation}

Measurements involved particle number fluxes, particle velocities and continuous-phase flow properties. Particle number fluxes were measured by collecting particles in the containers for timed intervals at the bottom of the test chamber. Experimental uncertainties of these measurements were dominated by finite sampling times which were selected to keep uncertainties ( $95 \%$ confidence) $<10 \%$.

Particle velocities were measured by particle tracking, which differed from Parthasarathy \& Faeth (1990). This involved illuminating the central region of the test tank with a light sheet ( $100 \mathrm{~mm}$ high and $25 \mathrm{~mm}$ thick) from a stroboscopic light source having a flash time of $1 \mu \mathrm{s}$ and operating at frequencies $500-1000 \mathrm{~Hz}$. The particle tracks were recorded with an open camera lens at a magnification of 0.7 . Particle velocities were in the range $3000-6000 \mathrm{~mm} / \mathrm{s}$ so that the light sheet essentially stopped the motion of the particles and yielded tracks having 10-20 images on the film. Velocities were found from the known flashing rate and the spacing between particle images on the film. Experimental uncertainties were dominated by sampling limitations over the size range of the particles: they are estimated to be $<5 \%(95 \%$ confidence).

A two-point, phase-discriminating laser velocimeter (LV), with one fixed channel at the center of the test chamber and a second channel that could be traversed in the streamwise and cross-stream directions, was used to measure air velocities. Discrimination to remove velocity signals due to particles followed Modarress et al. (1984), while the arrangement was identical to that of Parthasarathy \& Faeth (1990) (which should be consulted for details). The air was seeded with oil drops (nominal diameter of $1 \mu \mathrm{m}$ using a TSI model 9306 atomizer) to provide data rates of $5-10 \mathrm{kHz}$.

The time between valid air velocity signals was small, $100-200 \mu \mathrm{s}$, in comparison to Kolmogorov time scales of 11-32 ms and integral time scales of 1.8-5.8 s; therefore, the low-pass filtered analog outputs of the burst-counter signal processor were time-averaged, ignoring periods when particles were present, to find unbiased time-averaged gas velocities. Signals were collected in bursts of $16-32 \mathrm{~K}$ elements, for total sampling times on the order of $5 \mathrm{~min}$, in order to achieve stationary values with acceptable uncertainties. One-point temporal spectra were measured over a frequency range of $10^{-3}-10^{4} \mathrm{~Hz}$ using several subfrequency intervals (including appropriately longer sampling times to resolve low frequencies) to provide reasonable dynamic ranges, as discussed by Parthasarathy \& Faeth (1990).

Kolmogorov length scales were $0.44-0.74 \mathrm{~mm}$, which were comparable to the dimensions of the measuring volumes, and the flows were homogeneous; therefore, gradient bias errors were small. Experimental uncertainties ( $95 \%$ confidence) were as follows: mean streamwise and cross-stream velocities, $<10 \%$ and $<40 \%$; fluctuating streamwise and cross-stream velocities, $<15 \%$ and $<20 \%$; spatial correlations in the streamwise and cross-stream directions, $<15 \%$ and $<30 \%$; and streamwise and cross-stream temporal spectra, $<30 \%$ and $<40 \%$ for frequencies $<0.01 \mathrm{~Hz}$ and $<15 \%$ and $<25 \%$ for all other frequencies (except for the effects of step noise at the highest frequencies which will be discussed later). The present experimental uncertainties are similar to uncertainties for the particle/water flows. They are high in comparison to measurements in typical turbulent flows, however, due to the relatively low flow velocities (ca. $10 \mathrm{~mm} / \mathrm{s}$ ) and very high turbulence intensities (generally in excess of $100 \%$ ) of the homogeneous dilute dispersed flows.

\subsection{Test conditions}

Properties of the $0.5,1.0$ and $2.0 \mathrm{~mm}$ dia particles are summarized in table 1 . Particle size distributions were measured by Parthasarathy \& Faeth (1990): they were roughly Gaussian and had standard deviations of approx. $10 \%$ of the nominal particle diameter. Particle velocities in the region of the measurements were independent of particle number fluxes, however, they were lower than terminal velocities due to the limited available distance between the lowest screen and the test chamber. Particle Reynolds numbers were in the range 116-780, which is comparable to the range considered during the particle/water measurements. Rates of particle acceleration in the region of the measurements were used to evaluate particle drag. Similar to the earlier particle/water 
Table 1. Particle properties

\begin{tabular}{lccc}
\hline Nominal diameter $(\mathrm{mm})$ & 0.5 & 1.0 & 2.0 \\
\hline Diameter standard deviation $(\mathrm{mm})$ & 0.045 & 0.085 & 0.17 \\
Relative velocity (mm/s) & 3360 & 4970 & 5640 \\
Reynolds number & 116 & 344 & 780 \\
Drag coefficient & 1.03 & 0.64 & 0.47 \\
Wake momentum diameter $(\mathrm{mm})$ & 0.18 & 0.28 & 0.48 \\
\hline
\end{tabular}

${ }^{2}$ Round glass beads, of density $2450 \mathrm{~kg} / \mathrm{m}^{3}$, falling in stagnant air at $298 \pm 2 \mathrm{~K}$. Air properties: density $=1.17 \mathrm{~kg} / \mathrm{m}^{3}$, kinematic viscosity $=16.7 \times 10^{-6} \mathrm{~m}^{2} / \mathrm{s}$.

measurements, drag coefficients agreed within $15 \%$ of estimates based on the standard drag coefficient, $C_{\mathrm{D}}$, for spheres (Putnam 1961):

$$
C_{\mathrm{D}}=24\left(1+\operatorname{Re}^{2 / 3} / 6\right) / \operatorname{Re},
$$

where the particle Reynolds number is defined as

$$
\operatorname{Re}=U d / v
$$

and $U$ is the local mean relative velocity, $d$ is the nominal particle diameter and $v$ is the kinematic viscosity of air. These conditions yield wake momentum diameters, $\theta$, defined as

$$
\theta=\left(C_{\mathrm{D}} d^{2} / 8\right)^{1 / 2}
$$

in the range $0.18-0.48 \mathrm{~mm}$.

A range of particle number fluxes, $\dot{n}^{\prime \prime}$, for each particle size was considered for measurements of velocity fluctuations. The extremes of these conditions and a typical mid-range condition are summarized in table 2 . Mean particle spacings, $l_{p}$, were found assuming that the particles were falling randomly with a uniform particle number flux and relative velocity, as follows:

$$
l_{\mathrm{p}}=\left(U / \dot{n}^{\prime \prime}\right)^{1 / 3} \text {. }
$$

The resulting particle spacings were in the range $31-218 \mathrm{~mm}$, or $62-116$ particle diameters, yielding particle volume fractions $<0.0004 \%$.

Particle velocity fluctuations could not be measured accurately, however, estimates indicated that they were negligible due to modest air velocity fluctuations and poor particle response to air motion. Based on the particle/water study, the particle dispersing system did not introduce significant particle rotation. For such conditions, the rate of dissipation of turbulence kinetic energy within the measuring region, $\epsilon$, can be equated to the rate of generation of turbulence by particles. The rate of generation of turbulence is equal to the rate of loss of particle mechanical energy as they fall through the bath, i.e.

\begin{tabular}{|c|c|c|c|c|c|c|c|c|c|}
\hline \multirow[b]{2}{*}{ Loading } & \multicolumn{3}{|c|}{$d=0.55 \mathrm{~mm}$} & \multicolumn{3}{|c|}{$d=1.0 \mathrm{~mm}$} & \multicolumn{3}{|c|}{$d=2.0 \mathrm{~mm}$} \\
\hline & Low & Medium & High & Low & Medium & High & Low & Medium & High \\
\hline$\dot{n}^{\prime \prime}\left(\mathrm{kpart} / \mathrm{m}^{2} \mathrm{~s}\right)$ & 17.4 & 55.4 & 110.8 & 3.7 & 9.2 & 20.9 & 0.54 & 1.10 & 3.30 \\
\hline$l_{\mathrm{p}}(\mathrm{mm})$ & 57.8 & 39.3 & 31.2 & 110.4 & 81.5 & 62.0 & 218.0 & 172.5 & 119.6 \\
\hline$\epsilon\left(\mathrm{mm}^{2} / \mathrm{s}^{3}\right)$ & 20,400 & 64,900 & 129,900 & 26,300 & 65,300 & 148,300 & 16,300 & 33,000 & 98,000 \\
\hline$l_{\mathrm{K}}(\mathrm{mm})$ & 0.70 & 0.52 & 0.44 & 0.65 & 0.52 & 0.42 & 0.74 & 0.62 & 0.47 \\
\hline$t_{\mathrm{K}}(\mathrm{ms})$ & 29 & 16 & 11 & 25 & 16 & 11 & 32 & 23 & 13 \\
\hline$u_{\mathrm{K}}(\mathrm{mm} / \mathrm{s})$ & 24 & 32 & 39 & 26 & 32 & 40 & 23 & 27 & 36 \\
\hline$\left(\bar{u}^{\prime 2}\right)^{1 / 2}(\mathrm{~mm} / \mathrm{s})$ & 8.6 & 20.4 & - & 9.7 & 17.2 & 29.0 & 11.8 & 15.0 & 22.6 \\
\hline$\left(\bar{v}^{\prime 2}\right)^{1 / 2}(\mathrm{~mm} / \mathrm{s})$ & 8.8 & 13.5 & 18.5 & 2.4 & 6.0 & 12.1 & 8.4 & 7.9 & 13.0 \\
\hline $\bar{u}(\mathrm{~mm} / \mathrm{s})$ & 6.4 & 55.4 & - & 0.5 & 22.6 & 69.4 & 1.5 & 1.2 & 47.9 \\
\hline $\bar{v}(\mathrm{~mm} / \mathrm{s})$ & 2.8 & 8.6 & 8.9 & 0.9 & 2.6 & 6.4 & 1.7 & 1.1 & 2.8 \\
\hline$L_{u x}(\mathrm{~mm})$ & 117 & 98 & - & 123 & 97 & - & 143 & 176 & - \\
\hline$L_{u y}(\mathrm{~mm})$ & 40.1 & 38.2 & 一 & 12.5 & 20.0 & - & 24.9 & 23.2 & - \\
\hline$T_{v}(\mathbf{s})$ & 1.8 & - & - & 2.6 & - & - & 5.8 & - & - \\
\hline$T_{n}(\mathrm{~s})$ & 3.2 & - & - & 3.4 & - & - & - & - & - \\
\hline
\end{tabular}

$$
\epsilon=\pi \dot{n}^{\prime \prime} d^{2} C_{\mathrm{D}} U^{2} / 8
$$

Table 2. Summary of test condition

'Particle volume fractions $<0.0004 \%$, direct dissipation by particles $<1 \%$. 
Since particle velocities in the measuring region are known, however, it is more accurate to estimate the rate of turbulence generation as the rate of loss of potential energy of the particles as they fall through the bath, minus their rate of increase of kinetic energy, as follows:

$$
\epsilon=\pi \dot{n}^{\prime \prime} d^{3}\left[g\left(\rho_{\mathrm{p}}-\rho\right)-\rho_{\mathrm{p}} U \mathrm{~d} U / \mathrm{d} x\right] /(6 \rho),
$$

where $g$ is the acceleration of gravity, $\rho_{\mathrm{p}}$ and $\rho$ are the particle and air densities and $x$ is distance in the vertical (streamwise) direction. Due to the much lower density of air than water, rates of dissipation for the particle/air flows in table 2 are $10^{3}-10^{4}$ times larger than the earlier particle/water experiments. This was the main reason for the present interest in the particle/air flows because $\epsilon$ tends to dominate the flow properties.

The Kolmogorov length, $l_{\mathrm{K}}=\left(v^{2} / \epsilon\right)^{1 / 4}$, time, $t_{\mathrm{K}}=(v / \epsilon)^{1 / 2}$, and velocity $u_{\mathrm{K}}=(\epsilon v)^{1 / 4}$, scales also appear in table 2. The Kolmogorov length scales are comparable to the LV measuring volume and are somewhat smaller than the particle diameters. Measured air streamwise and cross-stream velocity fluctuations, $\left(\bar{u}^{\prime 2}\right)^{1 / 2}$ and $\left(\bar{v}^{\prime 2}\right)^{1 / 2}$, are comparable to the Kolmogorov velocity scales although $u_{\mathrm{K}}$ changes at a lower rate as $\epsilon$ is changed (similar to the particle/water flows, velocity fluctuations are proportional to $\epsilon^{1 / 2}$, while $u_{\mathrm{K}}$ is proportional to $\epsilon^{1 / 4}$ ). Mean velocities in the streamwise and cross-stream directions, $\bar{u}$ and $\bar{v}$, are comparable to velocity fluctuations. This behavior is similar to the particle/water experiments and is due to bulk motions caused by reduced particle fluxes near the surfaces of the particle distribution system and the walls of the test chamber. These mean motions preclude measurements of true temporal spectra at all but the lowest particle loadings, where means velocities are relatively small, but they do not affect the other measurements. Measured integral length scales, based on two-point correlations of streamwise velocity fluctuations in the streamwise and cross-stream dirctions, $L_{u x}$ and $L_{u y}$, are roughly twice those measured during the particle/water experiments. However, these length scales are still an order of magnitude smaller than the corresponding dimensions of the test chamber so that the surfaces of the apparatus has little effect on the continuous-phase turbulence properties. Temporal integral scales of velocity fluctuations in the streamwise and cross-stream directions, $T_{u}$ and $T_{v}$, are roughly two orders of magnitude smaller than sampling times so that the large-scale features of the flows are easily resolved.

In particle-laden flows, dissipation of turbulence consists of conventional dissipation by the continuous phase and turbulence modulation, i.e. direct dissipation by interactions between particle and continuous-phase velocity fluctuations. Parthasarathy \& Faeth (1990) show that the fraction of dissipation due to turbulence modulation is proportional to $U^{\prime 2} / U^{2}$, where $U^{\prime 2}$ is the variance of the relative velocity between the phases. As noted earlier, particle velocity fluctuations were small for the present conditions so that $\bar{U}^{\prime 2} \sim \bar{u}^{\prime 2}$ and the turbulence modulation portion of dissipation is proportional to the square of the relative turbulence intensity. For the present particle/air test conditions, the relative turbulence intensities were in the range $10^{-2}-10^{3}$ so that the effects of turbulence modulation were small, i.e. dissipation primarily occurred by processes within the continuous phase, similar to single-phase turbulent flows.

\section{THEORETICAL METHODS}

\subsection{Velocity fluctuations}

The simplified analysis described by Parthasarathy \& Faeth (1990) was used to help interpret and correlate the measurements. A stochastic method is used that explicitly considers the effects of individual particle flow fields, analogous to the approach of Batchelor (1972) for sedimentation processes. The major assumptions of the analysis are as follows: the flows are statistically stationary with uniform particle fluxes and constant continuous-phase properties; particle arrival times at an increment of area satisfy Poisson statistics, i.e. they are independent of other particle arrival times (Rice 1954); the flows are infinite in extent because the measurements were relatively independent of test chamber volume (Parthasarathy \& Faeth 1990); the flows are dilute so that the probability of a test point being within a particle is negligibly small; similarly, the contribution of flow properties immediately around the particles is ignored because it is small $(<10 \%)$ in comparison to the contributions of particle wakes due to much 
larger wake volumes (Parthasarathy \& Faeth 1990); and because the equations of motion are linear for asymptotic wakes, flow properties are taken to be the result of a linear superposition of particle flow fields that have reached the point of observation. The last assumption implies that the flow is sufficiently dilute so that distortion of each wake by other wakes can be ignored. This is questionable because velocity fluctuations were always present at the measuring volume, however, the approach was still pursued because it was helpful for the particle/water flows (Parthasarthy \& Faeth 1990).

Summing flow properties under these assumptions is an extension of methods used to analyze random noise (Rice 1954). Let the point of observation be the origin of a cylindrical coordinate system with $x$ being the streamwise direction and $r$ and $\phi$ the radial and azimuthal coordinates. Considering the potential effects of wake unsteadiness or turbulence, the arrival of a particle at $x=0, r, \phi$ and time $t=0$ can produce effects $g(r, \phi, t)$ and $g^{\prime}(r, \phi, t)$ at the point of observation due to the mean and turbulent wake properties. Following Rice (1954), Campbell's theorem can be extended to treat random arrivals of particles over a plane as a summation of effects of individual particles (Parthasarathy \& Faeth 1990). This yields the time-averaged effect,

$$
\bar{G}=\dot{n}^{\prime \prime} \int_{-\infty}^{\infty} \mathrm{d} t \int_{0}^{2 \pi} \mathrm{d} \phi \int_{0}^{\infty} g(r, \phi, t) r \mathrm{~d} r
$$

and the mean-squared fluctuation about the average,

$$
\bar{G}^{\prime 2}=\dot{n}^{\prime \prime} \int_{-\infty}^{\infty} \mathrm{d} t \int_{0}^{2 \pi} \mathrm{d} \phi \int_{0}^{\infty}\left[g^{2}(r, \phi, t)+g^{\prime 2}(r, \phi, t)\right] r \mathrm{~d} r .
$$

Equations [7] and [8] are for monodisperse particle flows. Under the assumption of linear superposition, however, if $\phi(d)$ is generic property for a particular particle diameter, then the mean value of $\phi$ becomes

$$
\bar{\phi}=\int_{0}^{\infty} \phi(d) \operatorname{PDF}(d) \mathrm{d} d,
$$

where $\operatorname{PDF}(d)$ is the probability density function of particle diameter.

A difficulty in applying [7]-[9] to find the present flow properties involves proper estimates of the particle effects, $g$ and $g^{\prime}$. In particular, particle Reynolds numbers are modest, in the range 38-780 for the combined particle/water and air measurements, and the particle wakes are within their self-generated turbulent field. There is no existing information concerning wake properties under these conditions and approximations must be made. Since wake Reynolds numbers are greater than unity in the region of interest, effects of turbulence in the asymptotic wake region cannot be ruled out ( Tennekes \& Lumley 1972). Therefore, integrations to find velocity fluctuations from [7]-[9] used mean velocity distributions for turbulent wakes from Tennekes \& Lumley (1972) and wake turbulence properties from Uberoi \& Freymuth (1970) in the integrations to find velocity fluctuations from [8] and [9], see Parthasarathy \& Faeth (1990) for the specific formulas.

A second problem encountered with integrations of [7]-[9], when using effects estimated from particles wakes, is that the integrals do not converge as $t \rightarrow \infty$. A similar difficulty is encountered for Stokes flow around particles during analysis of sedimentation, however, this can be resolved based on rigorous knowledge of Stokes flow (Batchelor 1972). Since information on wake properties in the present Reynolds number regime is empirical, and is questionable for the reasons noted earlier, a similar attack on the convergence problem would be premature. Instead, the integrations are terminated at $x / d=175$, which yields estimates of velocity fluctuations that are in reasonably good agreement with the measurements. Additionally, mean velocity defects in the wakes are comparable to levels of ambient velocity fluctuations at this location, suggesting that identifiable wake properties are lost in the background turbulent field in the region where the integrations are terminated. Finally, divergence of velocity fluctuations is relatively slow, proportional to $(x / d)^{1 / 6}$; therefore, the exact selection of the value of $x / d$ where integrations are ended is not very critical. 
Using turbulent asymptotic wake properties, and terminating integrations at $x / d=175$, yields the following expressions for the relative turbulence intensities in the streamwise and cross-stream directions (Parthasarathy \& Faeth 1990):

$$
\left(\bar{u}^{\prime 2}\right)^{1 / 2} / U=C_{u}\left[\epsilon d(\theta / d)^{2 / 3} / U^{3}\right]^{1 / 2}
$$

and

$$
\left(\bar{v}^{\prime 2}\right)^{1 / 2} / U=C_{v}\left[\epsilon d(\theta / d)^{2 / 3} / U^{3}\right]^{1 / 2},
$$

where $C_{u}=6.84$ and $C_{v}=4.63$. Using $C_{v}=C_{u} / 2=3.42$, however, provides a better fit of the measurements, as will be discussed later. The parameter $\epsilon d(\theta / d)^{2 / 3} / U^{3}$ on the right-hand sides (r.h.s.) of [10] and [11] appears later and will be called the dissipation factor for convenience.

\subsection{Correlations and spectra}

Expressions for correlations and spectra from the simplified theory can be found in Parthasarathy \& Faeth (1990). However, while the theory yielded reasonable estimates of velocity fluctuations, predictions of two-point spatial correlations and integral length scales were not very satisfactory. This was attributed to uncertainties about wake properties within the Reynolds number range and ambient turbulence levels of the present dispersed flows. In particular, the ambient flow was shown to deflect and distort the trajectory of the wake axis, which is likely to have a significant effect on spatial correlations and integral scales even though velocity scales might be influenced only to a modest degree. In view of these observations, an alternative empirical approach was used to treat correlations, spectra and integral scales. First of all, a direct result of the simplified theory (which agrees with experimental observations), is that correlation coefficients and normalized spectra should be independent of properties like $\dot{n}^{\prime \prime}$ and $\epsilon$. Additionally, particle size and Reynolds number did not have a significant effect on correlations and spectra so that assuming universal forms of these functions (determined by the measurements) seems reasonable. As a result, only generalized relationships for integral scales must be found.

Spatial integral scales are assumed to be proportional to dissipation length scales represented in the usual manner, i.e. $\sim k^{3 / 2} / \epsilon$ based on dimensional considerations, where $k$ is the turbulence kinetic energy. For the present dispersed flows, streamwise and cross-stream velocity fluctuations scale in the same manner from [10] and [11], orthogonal cross-stream velocity fluctuations are the same because the flows are homogeneous and no change in the degree of anisotropy of the flows was observed over the test range. This implies that integral scales, $L_{u x}$ and $L_{u y}$, should vary in the following manner:

$$
L_{u i} \epsilon / U^{3}=C_{u i}\left[\epsilon d(\theta / d)^{2 / 3} / U^{3}\right]^{3 / 2},
$$

where $i=x$ or $y$. The coefficients of proportionality, $C_{u i}$, are found by fitting the particle/water and air measurements. Additionally, the measurements do not confirm the $3 / 2$ power of the dissipation factor on the r.h.s. of [12] so this power was considered to be an empirical parameter as well.

Temporal integral scales are correlated by assuming that they were related to spatial integral scales and velocity fluctuations, i.e. $T_{i} \sim L_{u x} /\left(\bar{u}^{\prime 2}\right)^{1 / 2}$ based on dimensional considerations, where $i=u$ or $v$. Then, the expressions for the $L_{u x}$ and $\left(\bar{u}^{\prime 2}\right)^{1 / 2}$ from [10] and [12] yield the following correlating expressions for the temporal integral scales:

$$
T_{i} \epsilon / U^{2}=C_{T i}\left[\epsilon d(\theta / d)^{2 / 3} / U^{3}\right]
$$

where $C_{T i}$ is an empirical coefficient and $i=u$ or $v$. The $C_{T i}$ are found by correlating the measurements. Additionally, the measurements yield a power other than unity for the dissipation factor appearing on the r.h.s. of [13], as discussed later.

\section{RESULTS AND DISCUSSION}

\subsection{Evaluation of apparatus}

Apparatus evaluation during the particle/water study had shown that the apparatus was sufficiently large that wall effects had little influence on flow properties, e.g. reducing the test 
chamber volume by a factor of 32 had negligible effect on the measurements (Parthasarathy \& Faeth 1990). Thus, the present apparatus evaluation concentrated on establishing whether the flow was homogeneous because changes had been made in the particle distribution system.

The uniformity of particle number fluxes was tested by measuring the distribution of $\dot{n}^{\prime \prime}$ over the bottom of the chamber. Except for the region within $50 \mathrm{~mm}$ of the walls of the test chamber, the standard deviations of particle number fluxes at various positions were $<6 \%$ of the mean feed rate. This value was within experimental uncertainties so that $\dot{n}^{\prime \prime}$ was adequately uniform within the region of the present measurements. The spatial variation of r.m.s. streamwise velocity fluctuations was measured over the central portion of the test chamber $( \pm 120 \mathrm{~mm}$ in the cross-stream direction and $\pm 180 \mathrm{~mm}$ in the streamwise direction). The mean variations of velocity fluctuations over this region, from point-to-point and from test-to-test, were the same $(<10 \%)$. Thus, the velocity field within the region of the measurements also was homogeneous within experimental uncertainties.

\subsection{Velocity fluctuations}

The probability density functions of velocity fluctuations were Gaussian, similar to the particle/ water observations (Parthasarathy \& Faeth 1990); therefore, only the moments of the distribution are considered in the following. Measured relative turbulence intensities in the streamwise and cross-stream directions for both the particle/air and water tests are illustrated in figure 2 . The measurements are plotted according to the predictions of [10] and [11] with predictions based on the "best fit" results $\left(C_{u}=6.84\right.$ and $\left.C_{v}=C_{u} / 2\right)$ also shown on the plots.

Several properties of the measured velocity fluctuations in figure 2 agree with predictions and suggest that the wakes of individual particles make a significant contribution to continuous-phase properties. First of all, the degree of anisotropy is roughly 2, which is more representative of turbulent shear flows like particle wakes than the isotropic turbulence observed far from turbulence-generating grids (Hinze 1975). Next, for a given particle size and ambient fluid,

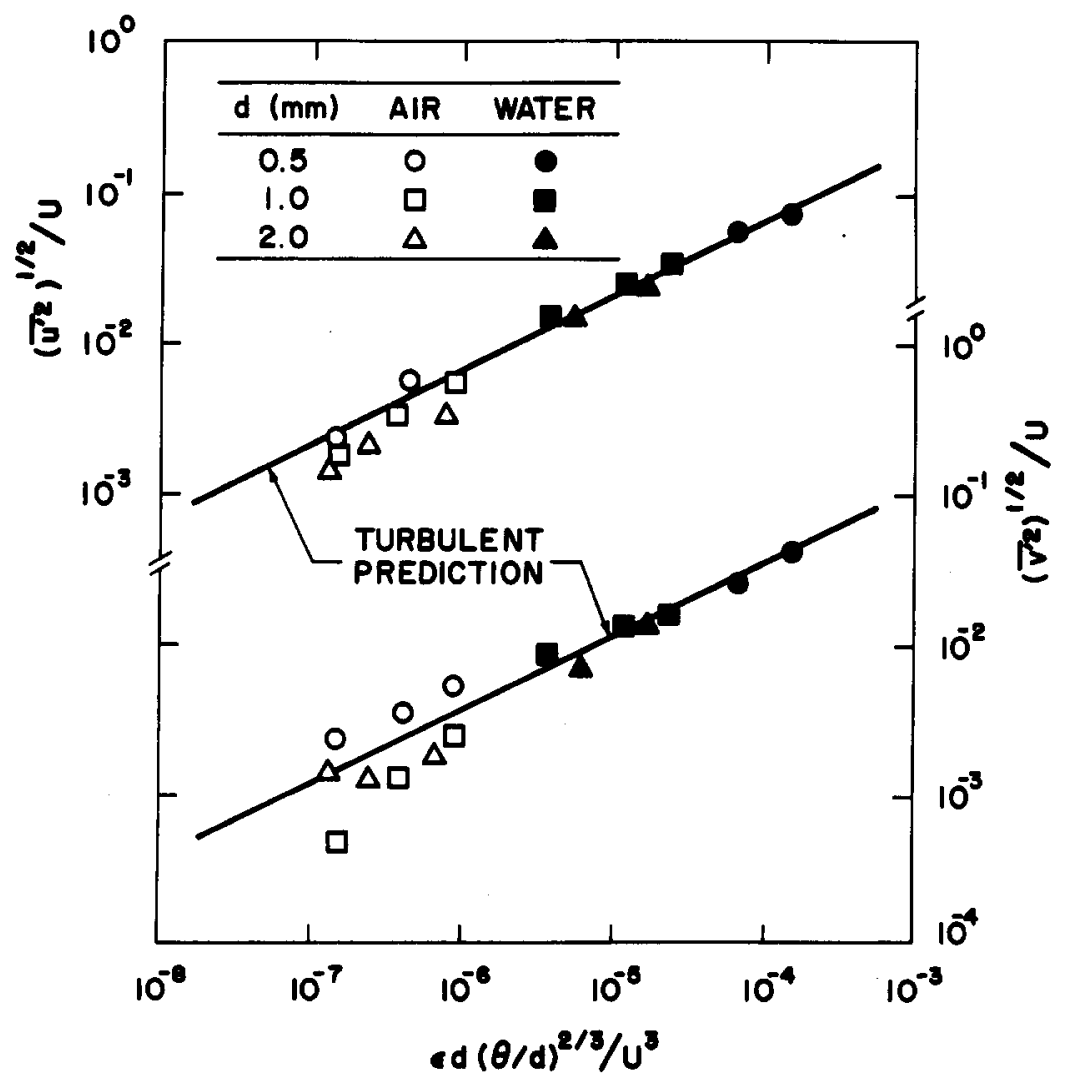

Figure 2. Streamwise and cross-stream r.m.s. velocity fluctuations. 
velocity fluctuations are proportional to $\epsilon^{1 / 2}$, and thus $\dot{n}^{\prime \prime 1 / 2}$ through [5] and [6] which agrees with theoretical predictions based on the summation of individual particle wake properties from [8]. Finally, the relative turbulence intensities correlate quite well as functions of the dissipation factor. In particular, the streamwise relative turbulence intensities agree with predictions based on turbulent wake properties for a reasonable integration limit $x / d=175$, as discussed earlier. The corresponding predictions of cross-stream relative turbulence intensity are not as satisfactory, however, yielding an anisotropy of 1.5 rather than the measured value of 2 .

The range of the correlations of relative turbulence intensities illustrated in figure 2 is relatively broad: relative turbulence intensities of $10^{-3}-10^{-1}$, dissipation factors of $10^{-7}-10^{-3}$ and particle Reynolds numbers of 38-780. Applying the correlations outside the range of the measurements, however, is questionable. First of all, conditions at higher relative turbulence intensities involve greater degrees of turbulence modulation than present test conditions so that increased dissipation by direct interaction between the particles and the continuous-phase turbulence might modify flow properties. Next, lower values of the dissipation factor than the present test range imply low levels of relative turbulence intensities, tending to reduce distortion and mixing rates of particle wakes by the ambient flow, with corresponding changes of continuous-phase turbulence properties. Finally, particle Reynolds numbers outside the present range should also modify wake properties. Lower Reynolds numbers approach sedimentation conditions that involve greater effects of the flow field around the particle in comparison to the wakes, and might better be treated using the approach of Batchelor (1972). Higher Reynolds numbers would yield more fully developed turbulent wakes, closer to the measurements of Uberoi \& Freymuth (1970), and modify continuous-phase turbulence properties accordingly.

\subsection{Spatial correlations}

Measured two-point correlation coefficients of streamwise velocity fluctuations in the crossstream and streamwise directions are illustrated in figures 3 and $\mathbf{4}$ for both the particle/air and water flows. In each case, the measurements are plotted as a function of streamwise or cross-stream distance, $x$ and $y$, normalized by the corresponding integral length scale. The measurements are

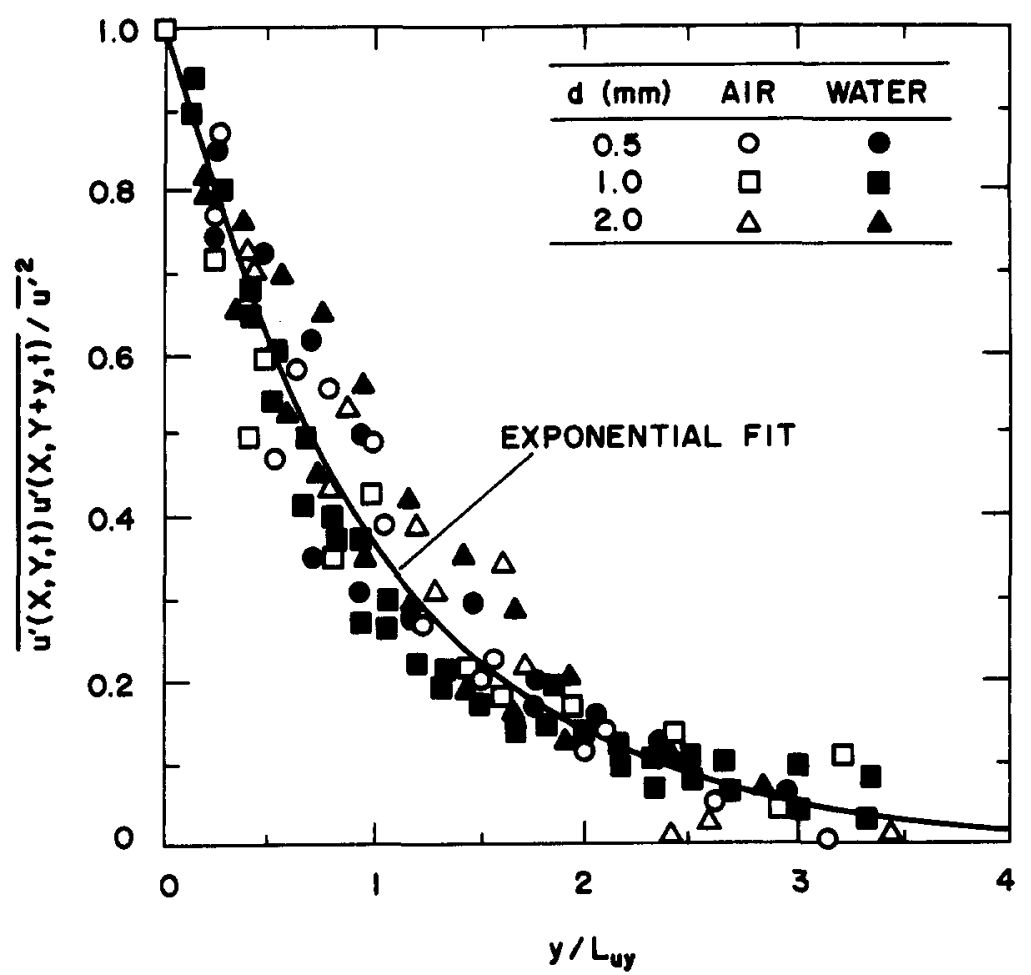

Figure 3. Spatial correlation of streamwise velocity fluctuations in the cross-stream direction for low and medium loadings. 


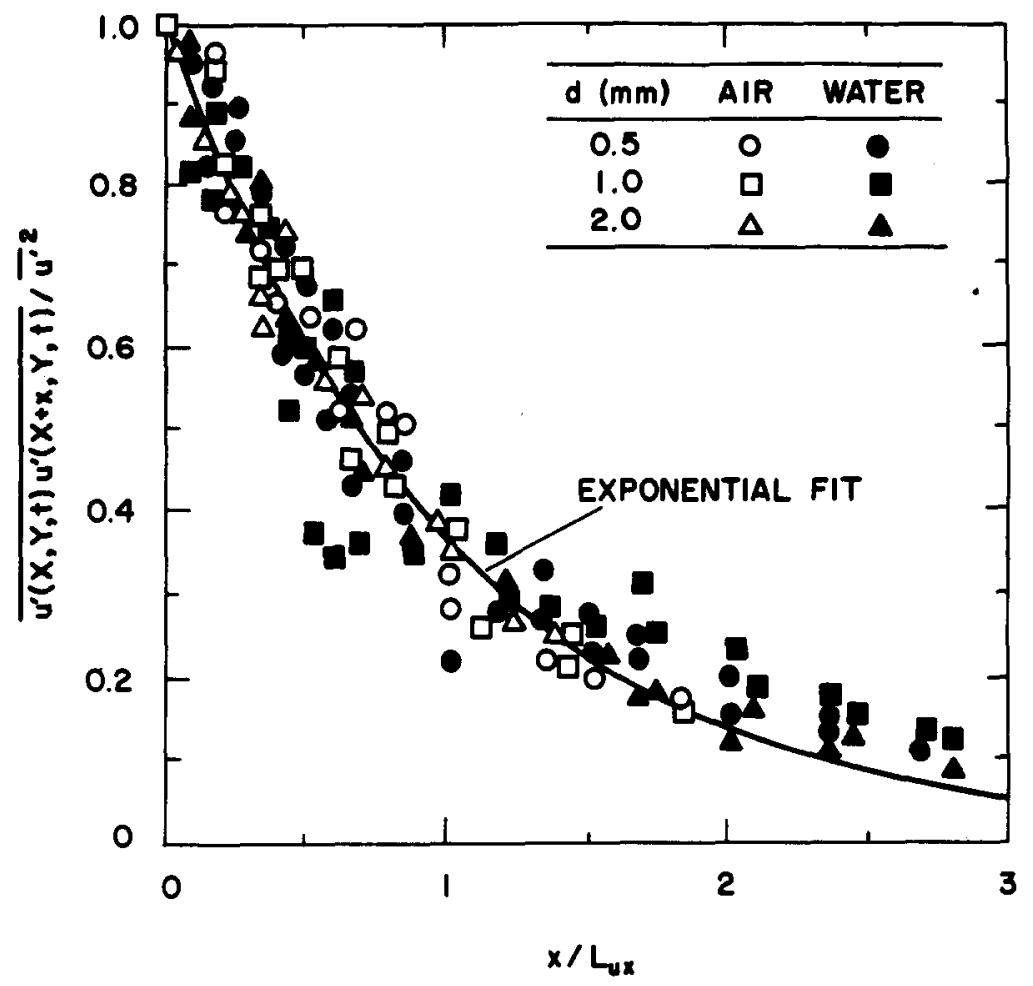

Figure 4. Spatial correlation of streamwise velocity fluctuations in the streamwise direction for low and medium loadings.

identified only according to particle size and continuous-phase fluid because particle number fluxes did not noticeably affect the correlations. Results only are shown for a single displacement direction, however, the correlations were symmetric within experimental uncertainties.

Ratios of Kolmogorov to integral length scales for the present dispersed flows are $<0.03$, and the Kolmogorov length scales are somewhat smaller than the LV measuring volumes; therefore, small-scale features near the origin are not captured by the results illustrated in figures 3 and 4 . Consistent with theory, the variation of correlation coefficients with normalized distance is independent of dissipation rate and the ambient fluid. The correlations are also independent of particle size. As noted earlier, predictions of correlations based on the velocity profiles of wakes were not very satisfactory, probably due to increased mixing rates and distortion of the wakes by continuous-phase turbulence. However, simple exponential functions, $\exp \left(-y / L_{u y}\right)$ and $\exp \left(-x / L_{u x}\right)$, are seen to provide reasonably good fits of the somewhat scattered data. An exception involves large separation distances for the correlation in the streamwise direction, where measured values are consistently higher than the exponential fits. Similar long tails of correlations in the streamwise direction were found from the predictions even though the predicted shapes of the correlations were not very satisfactory (Parthasarathy \& Faeth 1990). This suggests that the streamwise tails of the correlations are caused by the relatively large aspect ratios of wakes, analogous to the observed large levels of anisotropy.

Measured integral length scales in the streamwise and cross-stream directions for both the particle/air and water flows, are illustrated in figure 5. The dimensionless length scales are plotted as a function of the dissipation factor, as suggested by [12]. The following empirical fits of the measurements also are shown on the plots:

$$
L_{u i} \epsilon / U^{3}=C_{u i}\left[\epsilon d(\theta / d)^{2 / 3} / U^{3}\right]^{0.9} \text {, }
$$

where $i=y$ and $x$ and $C_{u y}=14$ and $C_{u x}=54$. Equations [14] provide a reasonable fit of the integral length scale measurements. The main exception involves $L_{u y}$ for the present particle/air measurements, where there is significant scatter about the fitted curve. However, these conditions also 


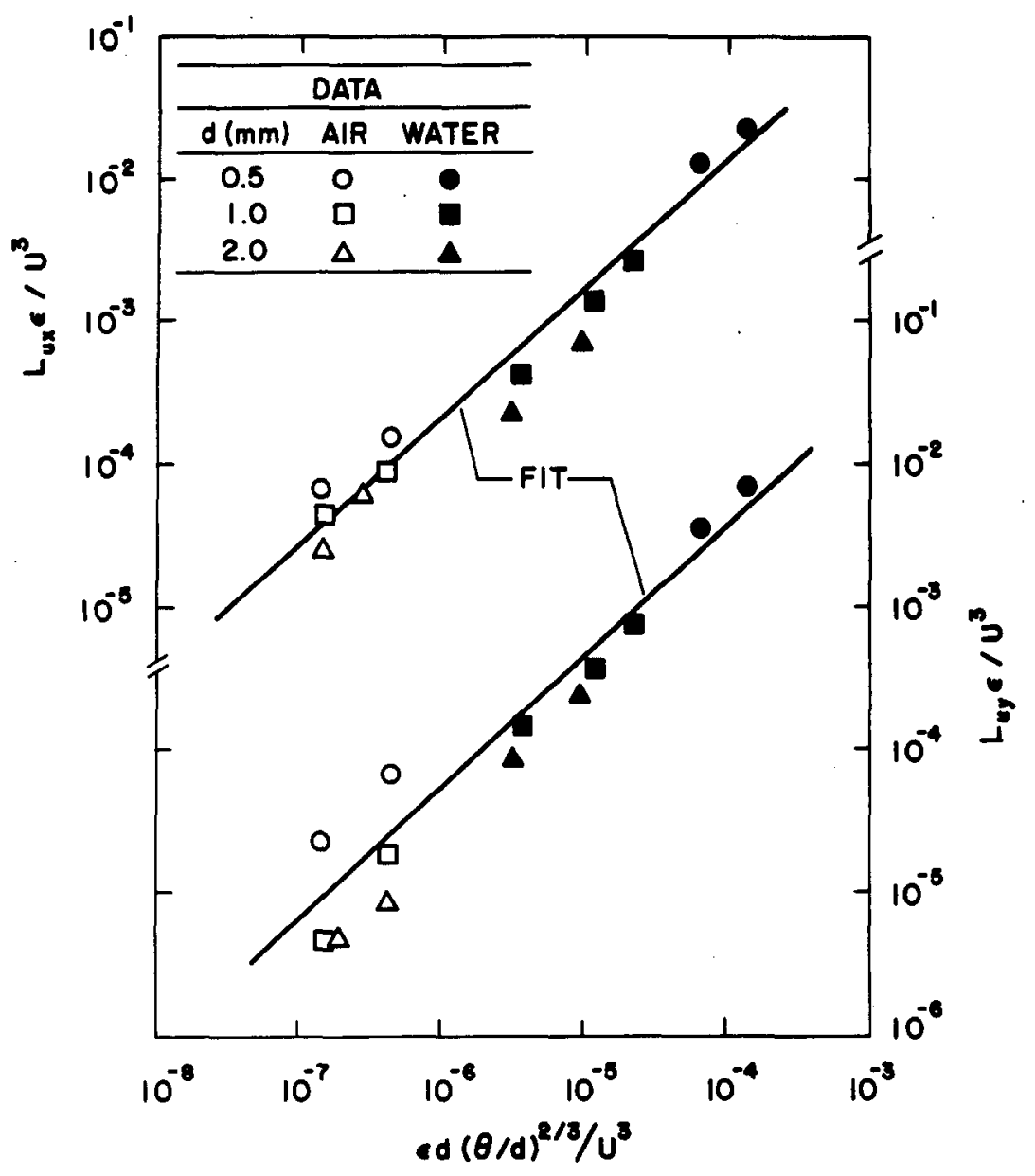

Figure 5. Streamwise and cross-stream spatial integral scales.

exhibit relatively large scatter for $\left(\bar{v}^{\prime 2}\right)^{1 / 2}$ in figure 2 , so that measurement difficulties due to the smaller value of cross-stream than streamwise velocity fluctuations may be the source of the problem. Additionally, some systematic effects of particle size can be seen, with results for the smallest and largest particles tending to be consistently above and below the fitted line, respectively. However, the degree of these variations is not significant in comparison to the experimental uncertainties of the measurements.

An interesting feature of the measurements in figure 5 is that the dimensionless length scales increase according to the 0.9 power of the dissipation factor. This is substantially lower than the $3 / 2$ power estimate of [12], which was based on the correspondence between integral and dissipation length scales. An explanation of the reduced effect of the dissipation factor on integral length scales is that high values of the dissipation factor imply large relative turbulence intensities within the flow (see figure 2). This tends to increase mixing rates and distortion of the wakes, and reduces the streamwise distance to the point where the velocity defect within the wakes becomes comparable to ambient velocity fluctuations. These effects should tend to reduce integral length scales, with corresponding reductions in the rate of increase of the length scales with increasing dissipation factor.

The integral length scales exhibit significant anistropy, with $L_{u x} / L_{u y}$ roughly 3.9 over the range of the measurements, which is typical of shear flows like wakes (Hinze 1975). Additionally, the fitted correlations for the length scales of [14] have a relatively weak dependence on $\epsilon$ and $U$. The particle property dependence is roughly proportional to $d C_{\mathrm{D}}^{2 / 3}$ which has not been varied to a great degree over the experiments (only a standard deviation of roughly one-third from the mean value of this parameter). This accounts for the modest changes of integral length scales over the present range of test conditions. 
The relatively large range of length scales of the continuous phase, in spite of relatively low particle Reynolds numbers, is an interesting feature of the present flows (Parthasarathy \& Faeth 1990). This can be quantified from [14] and the expression for the Kolmogorov length scale, as follows:

$$
L_{u i} / l_{K}=C_{u i}\left(U^{4} / \epsilon v\right)^{3 / 4}\left[\epsilon d(\theta / d)^{2 / 3} / U^{3}\right]^{0.9} .
$$

For both the particle/air and water flows, $L_{u x} / l_{\mathrm{K}}$ is in the range $150-300$, with $L_{u y} / l_{K}$ proportionately smaller according to the integral length scale anisotropy ratio. This relatively large range of length scales, in spite of low particle and wake Reynolds numbers, is caused by the contribution of mean velocities in particle wakes to flow properties because particle arrivals are random. Equation [15] implies that the length scale ratio increases with increasing $U, \epsilon$ and $d$; however, the rates of increase are relatively weak so that variations of $L_{u x} / l_{\mathrm{K}}$ are not large over the range of the particle/air and water experiments.

\subsection{Temporal spectra}

Measured temporal power spectral densities of streamwise and cross-stream velocity fluctuations, $E_{u}(f)$ and $E_{v}(f)$, are plotted as a function of frequency, $f$, in figures 6 and 7 . The spectra and the frequencies have been normalized by the corresponding velocity fluctuations and temporal integral scales. The measurements are for particle/air flows and are limited to the lowest loadings for each particle size so that effects of mean velocities are small. Conditions at the onset of step noise due to the sample-and-hold signals of the LV processor are marked on the plots, based on estimates from Adrian \& Yao (1987): measurements at higher frequencies should be ignored. Predicted spectra, ignoring contributions of wake turbulence that primarily appear beyond the step-noise limit, also are shown on the plots. Similar results for the particle/water flows can be found in Parthasarathy \& Faeth (1990); however, measurements and predictions for the particle/water and air flows are nearly the same.

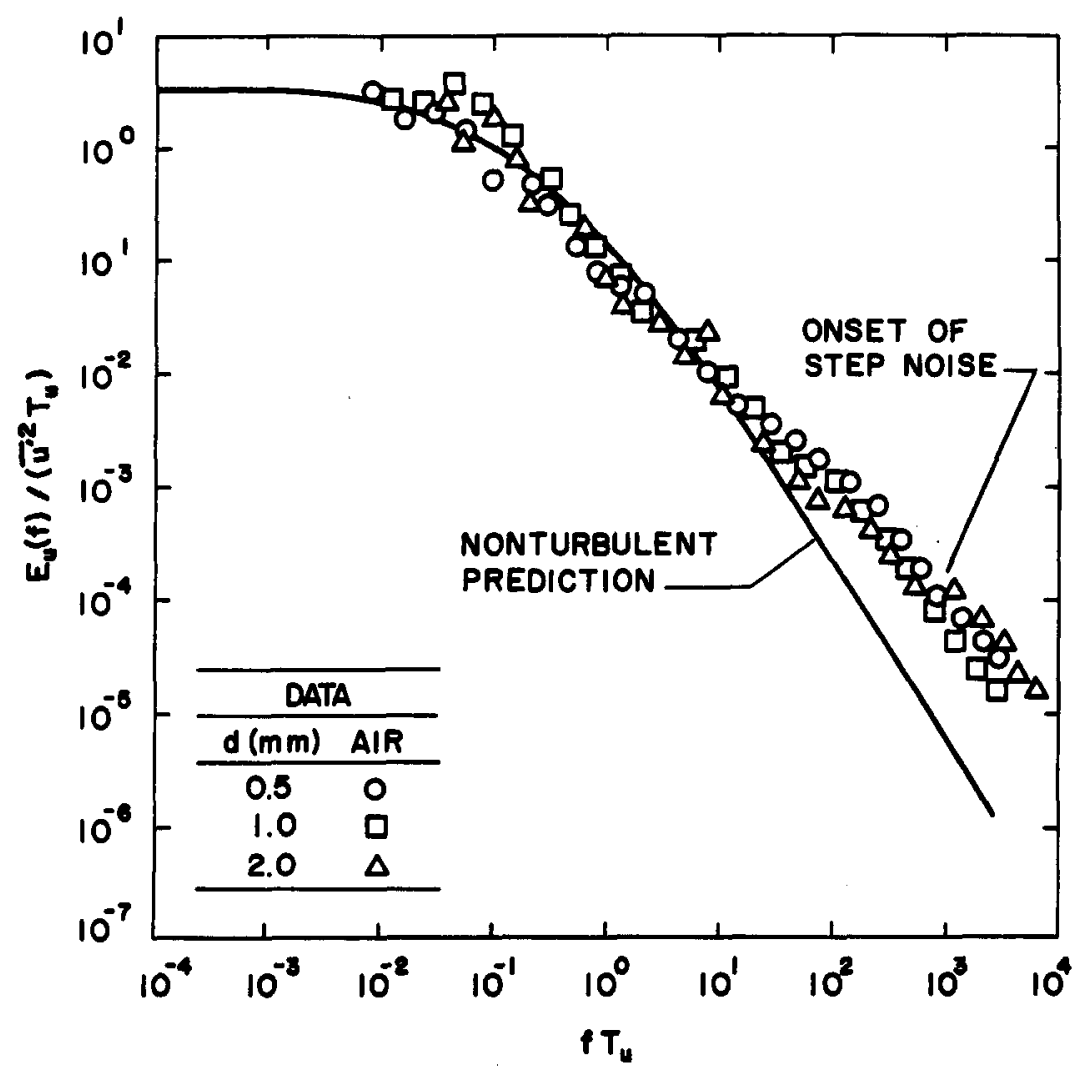

Figure 6. Temporal power spectral densities of streamwise velocity fluctuations. 


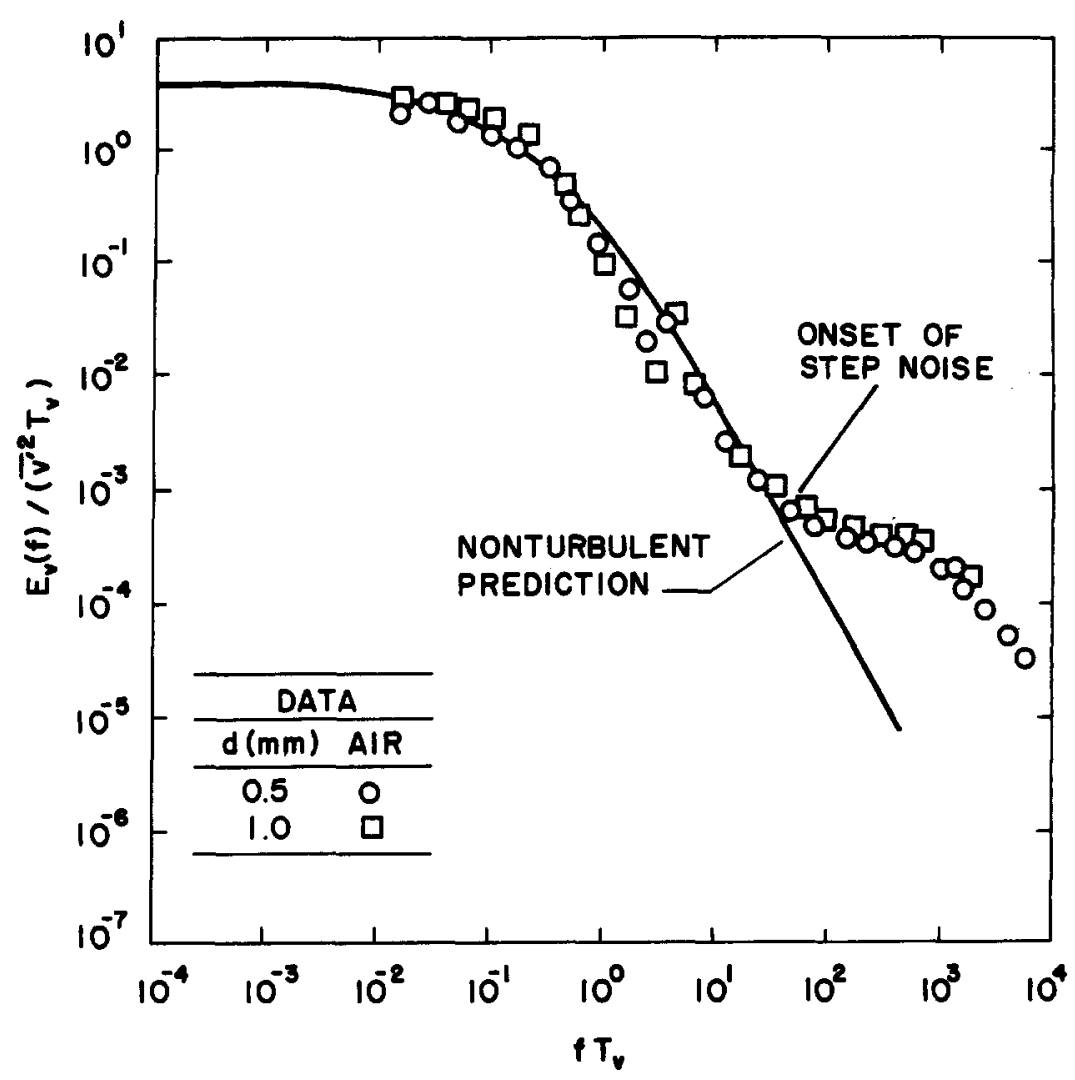

Figure 7. Temporal power spectra of cross-stream velocity fluctuations.

Effects of particle size and loading on the measured temporal spectra are small in comparison to experimental uncertainties. Dimensionless frequencies corresponding to the Kolmogorov microscale regime- $f T_{u}=T_{u} / t_{\mathrm{K}}$ and $f T_{v}=T_{v} / t_{\mathrm{K}}$-are in the range 60-180; unfortunately, measurements are not available in this region due to the intrusion of step noise. The spectra decay for dimensionless frequencies in the range $10^{-2}-10^{2}$. As noted earlier, the relatively large range of time scales for low wake Reynolds numbers is probably due to contributions from mean velocities in particle wakes. This view is supported by the properties of the inertial-like regions and spectral decay. Rather than decaying according to $f^{-5 / 3}$, typical of conventional turbulence, $E_{u}$ decays according to $f^{-1.1}$ and $E_{v}$ according to $f^{-1.5}$. The predictions based on mean velocity profiles in turbulent wakes, illustrated in figures 6 and 7, agree quite well with these trends. Even use of mean velocity profiles for laminar wakes yields similar behavior (Parthasarathy \& Faeth 1990). This lack of sensitivity to the shape of mean velocity profiles in the wakes suggests that effects of distortion of the wakes by ambient turbulence may not have a large influence on normalized spectra in the range of frequencies that could be resolved. Additionally, direct contributions of wake turbulence, if present, only appear for dimensionless frequencies beyond the step noise limit, as noted earlier. Taken together, the properties of the temporal spectra appear to be largely governed by mean velocities in randomly-arriving particle wakes.

Measured temporal integral scales of streamwise and cross-stream velocity fluctuations are illustrated in figure 8 for both the particle/air and water flows. The dimensionless time scales are plotted as a function of the dissipation factor, as suggested by [13]. The following empirical fit of the measurements also is shown on the plot:

$$
T_{i} \epsilon / U^{2}=C_{T i}\left[\epsilon d(\theta / d)^{2 / 3} / U^{3}\right]^{0.4},
$$

where $i=u$ and $v$ and $C_{T u}=C_{T v}=2.5$. The temporal integral scale measurements illustrated in figure 8 are limited and scattered, however, [16] provides a reasonable fit of the data. In this case, no particular trend with respect to particle size is observed. Similar to the correlation of length scales, the power of the dissipation factor is smaller than estimates based on the correspondence 


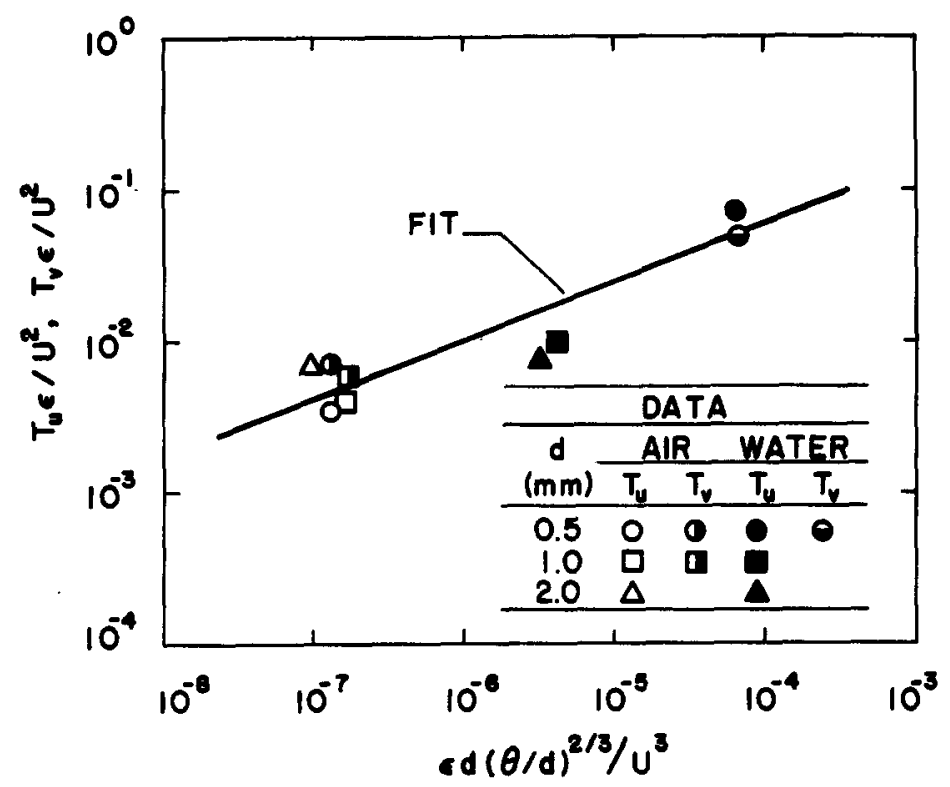

Figure 8. Temporal integral scales of streamwise and cross-stream velocity fluctuations.

between integral and dissipation length scales: 0.4 in [16] as opposed to 1.0 in [13]. Reasons for this behavior are analogous to those discussed in connection with the length scales.

The ratios, $T_{u} / t_{\mathrm{K}}$ and $T_{v} / t_{\mathrm{K}}$ were discussed earlier. Based on [16], and the expression for the Kolmogorov time scale, the functional forms of these ratios are as follows:

$$
T_{i} / t_{\mathrm{K}}=C_{T_{i}}\left(U^{4} / \epsilon v\right)^{1 / 2}\left[\epsilon d(\theta / d)^{2 / 3} / U^{3}\right]^{0.4} \text {. }
$$

Similar to the length scale ratios of [15], the time scale ratio increases with $U, \epsilon$ and $d$, but the rates of increase are relatively weak so that the variation of $T_{i} / t_{\mathrm{K}}$ is not large over the range of the particle/air and water experiments.

\section{CONCLUSIONS}

The present investigation considered the properties of particle-generated turbulence in the continuous phase of homogeneous dilute particle-laden flows. The specific configuration involved nearly monodisperse glass spheres falling in stagnant air, to supplement earlier findings for particles falling in stagnant water. The combined data base involved the following conditions: particle Reynolds numbers of 38-780; particle number fluxes of $0.54-110.8 \mathrm{kpart} / \mathrm{m}^{2} \mathrm{~s}$; mean particle spacings of $8.2-218 \mathrm{~mm}$; rates of dissipation of $27-148,300 \mathrm{~mm}^{2} / \mathrm{s}^{3}$, particle volume fractions $<0.01 \%$; and relative turbulence intensities of $10^{-3}-10^{-1}$. The major observations and conclusions of the study are as follows:

(a) Relative turbulence intensities and integral scales could be correlated as functions of the dissipation factor $\left[\epsilon d(\theta / d)^{2 / 3} / U^{3}\right]$. However, use of these correlations outside the present test range is not recommended: higher relative turbulence intensities would involve significant effects of turbulence modulation; lower relative turbulence intensities would reduce turbulent distortion of particle wakes; and particle wake properties vary with Reynolds number-all potentially modifying flow properties from the present findings.

(b) A number of features of particle-generated turbulence are similar to other homogeneous turbulent flows; in particular, the probability density functions of velocity fluctuations approximate Gaussian functions, and the large-scale features of spatial correlation coefficients and normalized temporal spectra are relatively independent of flow conditions when plotted in terms of normalized distances and frequencies. 
(c) However, a number of features of particle-generated turbulence are distinctly different from other turbulent flows, probably due to contributions from mean velocities in randomly-arriving particle wakes; for example, the degree of anisotropy of the flow is usually large $\left[\left(\bar{u}^{\prime 2}\right)^{1 / 2}=2\left(\bar{v}^{\prime 2}\right)^{1 / 2}\right]$, length scales correlate with wake properties and are essentially independent of the mean spacing between particles, and streamwise temporal spectra decay proportional to $f^{-1.1}$ rather than $f^{-5 / 3}$.

(d) A simplified model, based on linear superposition of randomly-arriving particle velocity fields, was helpful for interpreting and correlating many features of the flow. However, more information on the character of particle wakes at modest Reynolds numbers in turbulent environments is needed for quantitiative assessment of this approach and a better understanding of the mechanisms controlling continuous-phase turbulence properties in these flows.

Acknowledgements-This investigation was supported by the Air Force Office of Scientific Research, Grant No. AFOSR-89-0516, under the technical management of J. M. Tishkoff. The U.S. Government is authorized to reproduce and distribute copies of this paper for governmental purposes notwithstanding any copyright notation thereon.

\section{REFERENCES}

AdRIAN, R. J. \& YAO, C. S. 1987 Power spectra of fluid velocities measured by laser Doppler velocimetry. Expts Fluids 5, 17-28.

Al TAweel, A. M. \& LandaU, J. 1977 Turbulence modulation in two-phase jets. Int. J. Multiphase Flow 3, 341-351.

BATCHELOR, G. K. 1972 Sedimentation in a dilute dispersion of spheres. J. Fluid Mech. 52, 245-268.

FaEth, G. M. 1987 Mixing, transport and combustion in sprays. Prog. Energy Combust Sci 13, 293-345.

HiNZE, J. O. 1972 Turbulent fluid and particle interaction. Prog. Heat Mass Transfer 6, 433-452.

HINZE, J. O. 1975 Turbulence, pp. 556-565. McGraw-Hill, New York.

HuMPhreYs, W. J. 1964 Physics of the Air, pp. 279-281. Dover, New York.

LANCE, M. \& BATAILLE, J. 1982 Turbulence in the liquid phase of a bubbly air-water flow. Advances in Two-phase Flow \& Heat Transfer, Vol. 1, pp. 403-427. Martinus Nijhoff, The Hague.

Lance, M., Marie, J. L., Charnay, G. \& Bataille, J. 1980 Turbulence structure of cocurrent air-water bubbly flow. NUREG/CP-0014, Vol. 2, pp. 403-427. Nuclear Regulatory Commission, Washington, DC.

LANCE, M., Marie, J. L. \& Bataille, J. 1985 Homogeneous turbulence in bubbly flow. Publication FEP-29, pp. 117-124. ASME, New York.

Modarress, D., TaN, H. \& Elghobashi, S. 1984 Two-component LDA measurements in a two-phase turbulent jet. $A 1 A A J l$ 22, 624-630.

Parthasarathy, R. N. 1989 Homogeneous dilute turbulent paticle-laden water flow. Ph.D. Thesis, Univ. of Michigan, Ann Arbor.

Parthasarathy, R. N. \& FAETH, G. M. 1987 Structure of particle-laden turbulent water jets in still water. Int. J. Multiphase Flow 13, 699-716.

Parthasarathy, R. N. \& Faeth, G. M. 1990 Turbulence modulation in homogeneous particleladen flows; ibid. Turbulent dispersion of particles in self-generated homogeneous turbulence. J. Fluid Mech. 220, 485-537.

PutNam, A. 1961 Integrable form of droplet drag coefficient. Am. Rocket Soc. J. 31, 1467-1468.

RICE, S. O. 1954 Mathematical analysis of random noise. In Noise and Stochastic Processes. pp. 133-294. Dover, New York.

SUN, T.-Y. \& FAETH, G. M. 1986 Structure of turbulent bubbly jets-II. Phase property profiles. Int. J. Multiphase Flow 12, 115-124.

Tennekes, H. \& Lumley, J. L. 1972 A First Course in Turbulence, pp. 113-124. MIT Press, Cambridge, MA.

UBEROI, M. S. \& FREYMUTH, P. 1970 Turbulent energy balance and spectra of the axisymmetric wake. Phys. Fluids 13, 2205-2210. 